\title{
Direct Dark Matter Detection with the XENON and DARWIN Experiments
}

\author{
Alexander Kish*i \\ Physics Department, University of Zurich \\ E-mail: alexkish@physik.uzh.ch
}

The XENON1T detector, currently under construction at the Gran Sasso Underground Laboratory (LNGS) in Italy, is a dual-phase (liquid-gas) xenon time-projection chamber (TPC) for particle detection. It is the successor of XENON100, which reached its sensitivity goal with the last limits on spin-independent WIMP-nucleus interaction $\left(2 \times 10^{-45} \mathrm{~cm}^{2}\right.$ at $\left.55 \mathrm{GeV} / \mathrm{c}^{2}\right)$, the worldleading result at the time of publication. The construction of the water tank, to be employed as a shield against environmental radiation and as a Cerenkov muon veto, was completed at LNGS in 2013; most other subsystems are currently under construction. The total amount of xenon to be housed in the XENON1T cryostat is $3 \mathrm{t}$, with a fiducial mass of about $1 \mathrm{t}$. In order to detect the VUV scintillation light from particle interactions with the xenon target, two arrays of 3-inch Hamamatsu R11410 photomultiplier tubes will be installed on the top and bottom of the TPC. The assembly of the inner detector components is planned for late 2014, and the science goal is expected to be reached after two years of continuous operation by 2017 . The next step in the XENON dark matter search program is the XENONnT project. It will double the amount of xenon in the sensitive volume $(\sim 6 \mathrm{t})$, which would allow to fiducialize the target to $\sim 4 \mathrm{t}$. The XENONnT TPC with the inner cryostat vessel will be constructed while XENON1T is taking data, and will be installed in the same outer vessel and the water shield as XENON1T. The exploration of the entire experimentally accessible WIMP parameter space, down to a region where solar neutrino interactions become an irreducible background (and eventually provide a possibility to precisely measure their low-energy spectrum in real-time), is foreseen with an ultimate experiment at the $20 \mathrm{t}$ scale. The design and R\&D works for such a project were initiated by the DARWIN (DARk matter WImp search with Noble liquids) consortium. In this publication, the current status and the plans of the XENON collaboration are presented, with focus on the design details of the XENON1T experiment, as well as on the future multi-ton DARWIN project.

Technology and Instrumentation in Particle Physics 2014,

2-6 June, 2014

Amsterdam, the Netherlands

\footnotetext{
* Speaker.

†n behalf of the XENON collaboration and the DARWIN consortium.
} 


\section{Particle Detection with a Dual-Phase Xenon Time-Projection Chamber}

The XENON project is dedicated to direct detection of dark matter in the form of WIMPs via their scattering off target nuclei in a dual-phase xenon detector. A schematic description of a particle detection in such a detector is shown in Fig. 1 [1]. When a particle interacts with a xenon atom, the energy transfer is split between ionization, excitation and heat [2,3]. An excited atom combines with another atom and produces an excited diatomic molecule (eq. 1). In the subsequent de-excitation it releases a photon with a wavelength of $178 \mathrm{~nm}$, in the vacuum ultra-violet (VUV) region (eq. 2). This prompt initial scintillation light (denoted S1) is detected by two arrays of photomultiplier tubes (PMTs) on the top and bottom of the target volume. Xenon atoms do not absorb their own scintillation light, because the photons originate from the decay of the excimer state. In the absence of an electric field, some of the electron-ion pairs produced by ionization recombine (eq. 4), and VUV photons are also emitted through an excimer (eq. 6) when it decays back top the ground state (eq. 7). However, when an electric field is applied across the liquid xenon target, some of the ionization electrons are removed from an interaction site, hence do not recombine with ions and can be detected independently from the S1 light signal. These electrons are drifted upwards and extracted into the gas phase above the liquid xenon target, and accelerated with a high electric field, producing an electroluminescence signal (S2) [4] via collisions with xenon atoms, which is detected by the PMTs. In addition, some of the energy is deposited in a non-radiative transition (eq. 5).

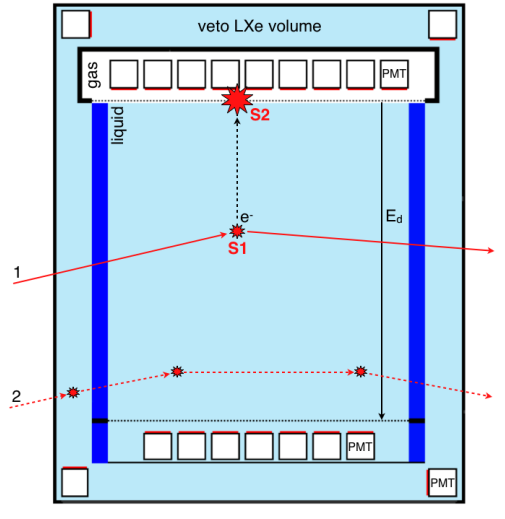

(a)

$$
\begin{gathered}
\mathrm{Xe}^{*}+\mathrm{Xe} \rightarrow \mathrm{Xe}_{2}^{*}, \\
\mathrm{Xe}_{2}^{*} \rightarrow 2 \mathrm{Xe}+h \nu \\
\mathrm{Xe}^{+}+\mathrm{Xe} \rightarrow \mathrm{Xe}_{2}^{+}, \\
\mathrm{Xe}_{2}^{+}+e^{-} \rightarrow \mathrm{Xe}^{* *}+\mathrm{Xe}, \\
\mathrm{Xe}^{* *} \rightarrow \mathrm{Xe}^{*}+\text { heat, } \\
\mathrm{Xe}^{*}+\mathrm{Xe} \rightarrow \mathrm{Xe}_{2}^{*}, \\
\mathrm{Xe}_{2}^{*} \rightarrow 2 \mathrm{Xe}+h \nu
\end{gathered}
$$

(b)

Figure 1: (a) - particle detection principle with a dual-phase TPC with an active LXe veto layer around the target: 1 - valid signal event, 2 - multiple scattering event with an interaction in the liquid xenon veto volume. (b) - physical processes in particle interaction with a xenon target. Figures from [1]

In the standard scenario, WIMPs are expected to elastically scatter off xenon nuclei resulting in low energy nuclear recoils (NR). Neutrons with energies in the MeV range passing through the detector also produce low energy nuclear recoils, whereas $\gamma$-rays and electrons produce electronic recoils (ER). Because of the different $d E / d x$, and hence different amount of recombination, the ratio of the $\mathrm{S} 1$ and $\mathrm{S} 2$ signals provides a possibility to distinguish ERs from NRs, and to reject the electromagnetic background with efficiency better than $99 \%$ at $\sim 50 \%$ NR acceptance [5, 6, 7].

In a homogeneous electric field, the position in the $X Y$ plane at which the proportional scintil- 
lation occurs is correlated with that of the original interaction, and leads to a clustered hit pattern on the top PMT array. This is used to reconstruct the $X$ and $Y$ coordinates of an event with a precision better than $3 \mathrm{~mm}(1 \sigma)$ [8] using pattern identification algorithms, which can be based on $\chi^{2}$-minimization, Support Vector Machines, or Neural Network methods. In addition, the time difference between the S1 and S2 signals provides information about the $Z$ coordinate of the interaction. The 3D position reconstruction capability allows localization and rejection of the events at the edges of the target volume, thus significantly reducing the external gamma and neutron backgrounds.

Optionally, the liquid xenon target can be surrounded from all sides by a liquid xenon layer, equipped with PMTs and acting as an active veto. Events that have a coincident signal in the veto (illustrated in Fig. 1) are removed from the analysis, which provides a significant reduction of background due to $\gamma$-interactions.

\section{The XENON100 detector for dark matter searches}

The current stage of the research project is the XENON100 detector, which is installed in the Laboratori Nazionali del Gran Sasso (LNGS), Italy. It is the successor of XENON10 [9], which has set some of the best limits on WIMP-nucleon inelastic scattering cross sections $[5,10]$. XENON100 improved this sensitivity by an increase of the target mass and a significant reduction of the background in the target volume, and by using an innovative design with a careful selection of the contraction materials, setting the limit of $2 \times 10^{-45} \mathrm{~cm}^{2}$ at $55 \mathrm{GeV} / \mathrm{c}^{2}$ [7], the world-leading result at the time of publication.

The passive shield of the XENON100 detector is shown in Fig. 2(a). It encloses the detector in $4 \pi$, and is installed on a thick slab of polyethylene. From outside to inside, it consists of tanks filled with water (Fig. 2(b)) to shield against ambient neutrons, placed on three sides and on top of the shield. Inside the water shield, there are two layers of lead (the inner one with a very low contamination of the radioactive isotope ${ }^{210} \mathrm{~Pb}$ [11]). Inside the lead, there is polyethylene to shield against further neutron backgrounds. The innermost shield layer consists of copper, which reduces the gamma background from the outer shield layers. The inner shield cavity is constantly purged with high purity boil-off nitrogen at a rate of $\sim 17$ standard liters per minute (slpm) in order to avoid penetration of radon.

The schematic drawing of the XENON100 detector is shown in Fig. 3(a). A total amount of $161 \mathrm{~kg}$ of LXe is enclosed in the vacuum insulated cryostat, made from low activity stainless steel of type 1.4571/316Ti. The quasi-cylindrical TPC is formed by 24 interlocking PTFE panels, see also Fig. 3(b). PTFE reflects scintillation light with high efficiency [12], and optically separates the $62 \mathrm{~kg}$ target volume from the surrounding liquid xenon, which is in average $4 \mathrm{~cm}$ thick, has a total mass of $99 \mathrm{~kg}$, and is lined on the walls and the bottom with a $1.5 \mathrm{~mm}$ thick PTFE layer.

Electrons created by ionization in the LXe target are drifted upwards by an electric field created by applying voltage on the cathode, a $75 \mu \mathrm{m}$ thick stainless steel mesh with hexagonal structure with a $5 \mathrm{~mm}$ pitch, installed on the bottom of the TPC. The cathode operation voltage is $-16 \mathrm{kV}$, which corresponds to a drift field across the TPC of $0.53 \mathrm{kV} / \mathrm{cm}$. In order to shield the bottom PMTs from this electric field, an additional grounded electrode (50 $\mu \mathrm{m}$ mesh) is installed below the cathode. 


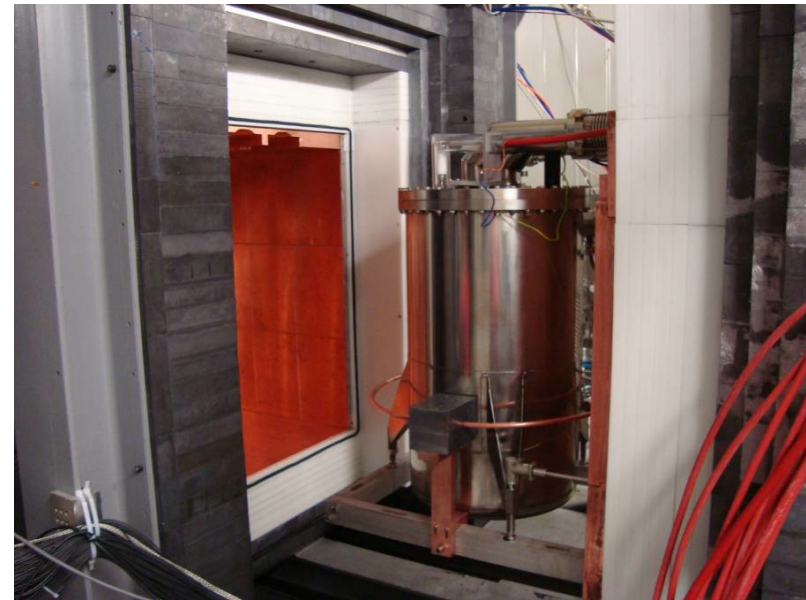

(a)

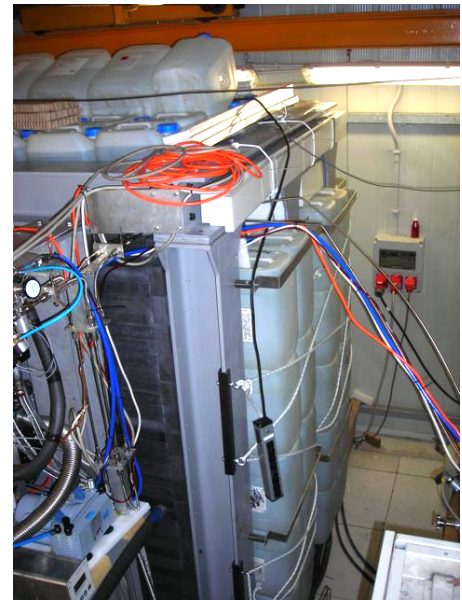

(b)

Figure 2: The XENON100 detector with the open shield door (a), and the water shield (b). Figures from [1].

The gas phase for charge amplification via proportional scintillation is maintained using a 'diving bell' system. It allows the liquid level to be kept constant at a precise height while having an additional layer of LXe above the TPC. A slight overpressure in the bell is provided by the gas returning from the continuous recirculation system. The liquid level is adjusted by changing the recirculation rate, and by adjusting the height of the gas outlet from the bell by a motion

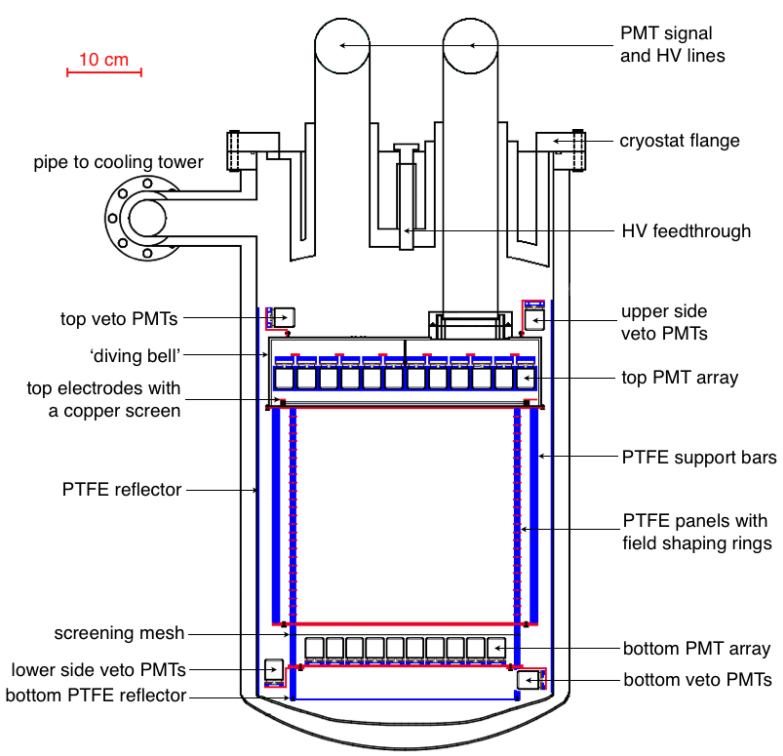

(a)

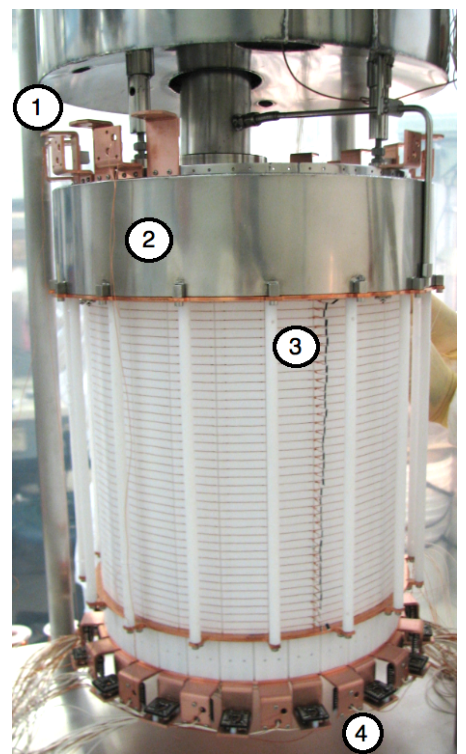

(b)

Figure 3: (a) - schematic view of the XENON100 detector. The colors show: black - stainless steel, red - copper, blue - PTFE. Figure published in [8]. (b) - the XENON100 TPC during assembly in the clean room: 1 - copper angles for the top and upper side veto PMTs, 2 - 'diving bell', 3 - resistor chain connecting the field shaping rings, 4 - copper holders for the bottom and lower side veto PMTs. Figure from [1] 
feedthrough.

An extraction field is created across the liquid-gas interface by applying high voltage on the anode, $125 \mu \mathrm{m}$ mesh with $2.5 \mathrm{~mm}$ pitch, which is placed inside the diving bell. This field is high enough to obtain an extraction efficiency close to $100 \%$ [13]. The entire stack of the top electrodes is optimized for optical transparency and minimal impact on the S2 resolution.

The scintillation light is detected by 242 one square inch R8520-06-AL Hamamatsu PMTs, optimized for radioactive contamination and for operation in LXe. The top PMT array consists of 98 PMTs in a PTFE support structure inside the diving bell, and 80 PMTs are installed below the cathode in the LXe. Additionally, 64 PMTs are mounted on copper angles and view liquid xenon of the veto volume.

\section{The XENON1T Experiment}

The new result on WIMP-nucleon scattering cross section from the LUX collaboration with a minimum at $7.6 \times 10^{-46} \mathrm{~cm}^{2}$ at $33 \mathrm{GeV} / \mathrm{c}^{2}$ [14], and theoretical predictions beyond the current limits $[15,16,17]$, impose that larger detectors with a lower radioactive background are necessary to continue the search for dark matter. One of such projects is the XENON1T detector, a dual-phase LXe TPC with a total mass of $\sim 3 \mathrm{t}$ and a fiducial mass of $\sim 1 \mathrm{t}$. The designed background level is below 1 event in $2 \mathrm{t}$-years exposure, leading to a sensitivity of $2 \times 10^{-47} \mathrm{~cm}^{2}$ for a $100 \mathrm{GeV} / \mathrm{c}^{2}$ WIMP.

In order to reduce the ambient backgrounds to negligible levels, the XENON1T detector will be placed inside a large water shield of $10 \mathrm{~m}$ height and $9.6 \mathrm{~m}$ diameter, instrumented with 84 8-inch Hamamatsu R5912 PMTs which provide an active Cerenkov veto [18]. The water tank has already been assembled in Hall B at LNGS, and the service building is being erected. The artistic rendering of the XENON1T detector in its water shield is shown in Fig. 4.

The core of XENON1T is a cylindrical TPC of $970 \mathrm{~mm}$ height and $960 \mathrm{~mm}$ diameter in a double-walled 316Ti stainless steel cryostat (hosting $\sim 2 \mathrm{t}$ of sensitive LXe; see Fig. 5(a)), suspended from a support frame which allows for the leveling within $100 \mu \mathrm{m}$ precision. In order to

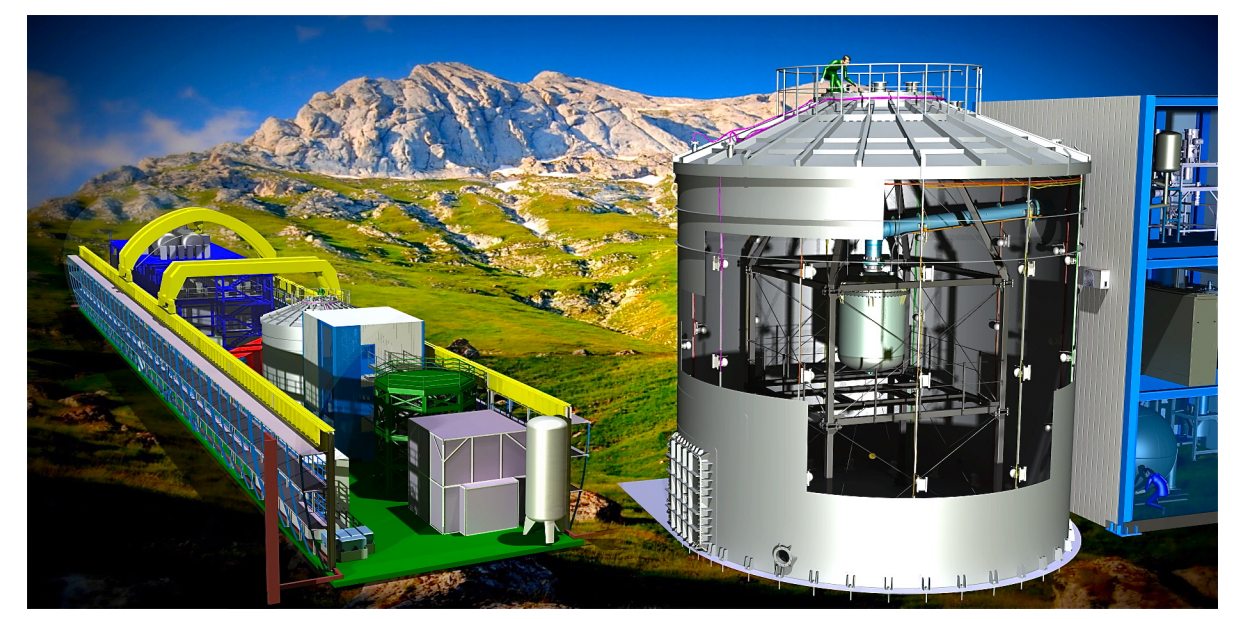

Figure 4: Artistic rendering of the XENON1T experiment in Hall B at LNGS (left), and the cryostat model inside the water shield (right). 


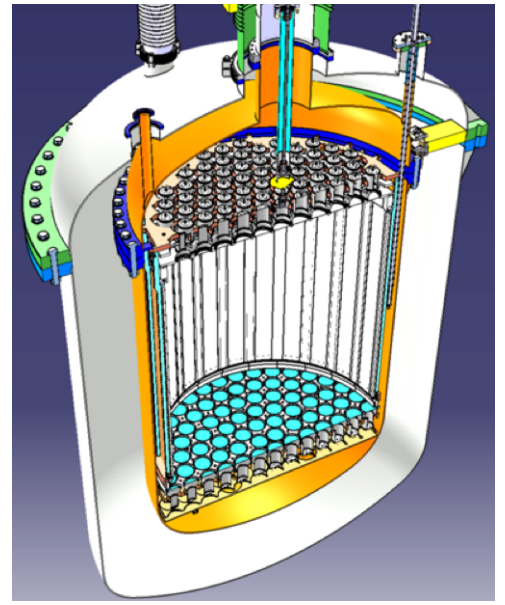

(a)

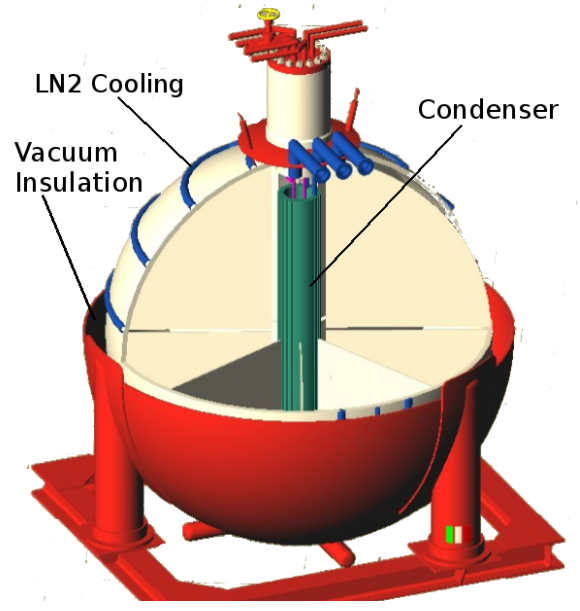

(b)

Figure 5: (a) - CAD model of the XENON1T TPC. The outer cryostat vessel is large enough to host the XENONnT TPC with double the amount of liquid xenon. (b) - a model of the high-pressure liquid xenon storage vessel ReStoX, which has been developed for the ton-scale XENON program, and can hold up to $7 \mathrm{t}$ of xenon in liquid or gaseous state.

achieve a drift field of $1 \mathrm{kV} / \mathrm{cm}$, the cathode at the bottom of the TPC will be biased with $-100 \mathrm{kV}$. The field homogeneity will be insured by 74 massive field shaping rings made out of OFHC copper. The prototypes of the grid and wire electrodes are being built, and a custom-made cathode high-voltage feedthrough was successfully tested for voltages of $-100 \mathrm{kV}$. The scintillation light from particle interactions will be detected by 3-inch R11410 Hamamatsu PMTs [19], arranged in two arrays (248 photosensors in total).

The XENON1T TPC is surrounded by a LXe layer, employed as an ultra-pure insulator between the TPC field cage and the cryostat vessel, and additionally providing the shield against extrinsic backgrounds. Hence, more than $3 \mathrm{t}$ of xenon is required to fill the detector. The xenon will be liquified by two redundant PTRs [20], each providing $200 \mathrm{~W}$ of cooling power, and installed at the end of the long umbilical pipe connecting the detector with the service building; an emergency cooling system is liquid nitrogen-based. The xenon will be stored in liquid phase in a dedicated 'ReStoX' system (Fig. 5(b)). The spherical vessel with active cooling can hold up to $7 \mathrm{t}$ of xenon, large enough for the anticipated upgrade to XENONnT. As the total designed heat load of the XENON1T is $<50 \mathrm{~W}$, the cooling system is also capable of servicing a larger detector.

\section{DARWIN Project}

DARk matter WImp search with Noble liquids (DARWIN) is an initiative to build the ultimate, multi-ton dark matter detector [21]. The project unites the ample expertise in Europe on liquid noble gas detectors, low-background techniques, cryogenic and underground infrastructures and on the physics issues related to direct dark matter and neutrino detection.

The primary goal of DARWIN is to probe the spin-independent WIMP-nucleon scattering cross section for $\sim 50 \mathrm{GeV} / \mathrm{c}^{2}$ WIMPs down to $10^{-49} \mathrm{~cm}^{2}$, where irreducible neutrino backgrounds set in. Two other major physics goals are the search for the neutrinoless double beta decay of 


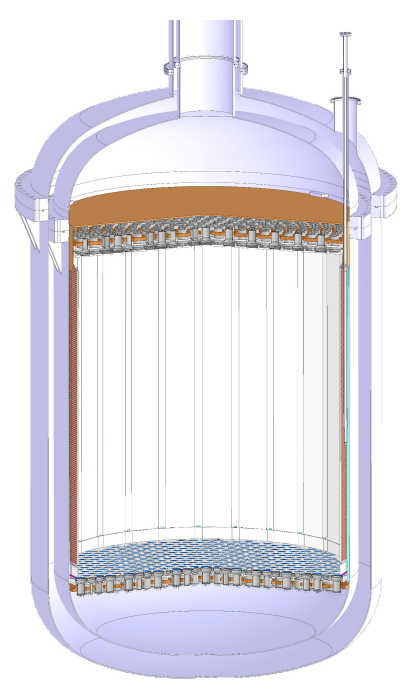

(a)

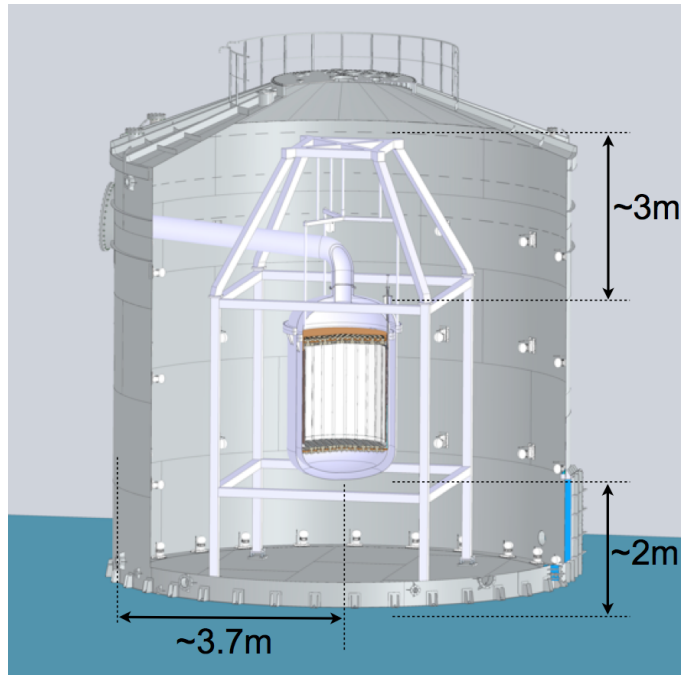

(b)

Figure 6: (a) - CAD model of the DARWIN detector. The drift length is $2.1 \mathrm{~m}$, the total amount of LXe is $21 \mathrm{t}$. (b) - DARWIN cryostat in the water tank of XENON1T/XENONnT.

${ }^{136} \mathrm{Xe}$ and the first real-time observation of solar pp-neutrinos with high statistics. The pp- and ${ }^{7} \mathrm{Be}-$ neutrinos together account for more than $98 \%$ of the total neutrino flux, and the measurement of ppneutrinos would test the main heat production mechanism in the Sun. The most robust predictions of the Standard Solar Model are for the pp-neutrino flux, which is heavily constrained by the solar luminosity, and an accurate measurement of the pp-neutrino flux will allow to distinguish between different neutrino oscillation scenarios.

In its baseline scenario, DARWIN will consist of multi-ton xenon-based, and possibly argonbased TPCs. The xenon-based part of the project could operate a cylindrical $\sim 2.1 \mathrm{~m} \times 2.1 \mathrm{~m}$ liquid xenon time-projection chamber (TPC) in a low-background cryostat (shown in Fig. 6(a)), installed in the water shield of the XENON1T and XENONnT experiments at LNGS (see Fig. 6(b)). Improved external shielding is possible by adding a liquid scintillator veto shield around the cryostat inside the water. The primary scintillation (S1), as well as the proportional scintillation signal from the charge (S2) are detected by two arrays of photosensors installed above and below the liquid xenon target, the most prominent candidate for which is 3-inch R11410 Hamamatsu PMT [19].

A detailed Monte Carlo study of the DARWIN sensitivity (the xenon-based detector) to WIMPs, to low-energy solar neutrinos, to coherent neutrino-nucleus scattering and to neutrinoless double beta decay has been performed [22], taking into account radioactive contamination in all the components, including the contributions from the intrinsic sources. The intrinsic background to xenon as a detection medium poses strong requirements on internal radio-activity levels: a contamination of the liquid xenon with natural krypton of about $0.1 \mathrm{ppt}$ and a radon level in the liquid of about $0.1 \mu \mathrm{Bq} / \mathrm{kg}$ are to be ensured. This can be achieved by purifying the noble gas (by isotope separation with a gas centrifuge, by distillation method, and by ultra-clean, charcoal-based radon filters), and by the use of materials with low radon emanation. 


\section{Summary}

The question on the nature of the dark matter, which is so far been observed only by its gravitational effects, is central to cosmology and particle physics. As shown in Fig. 7, the direct WIMP searches are led by experiments based on noble gases, but the theoretical parameter space is still not covered, and some new experiments are proposed and being constructed.

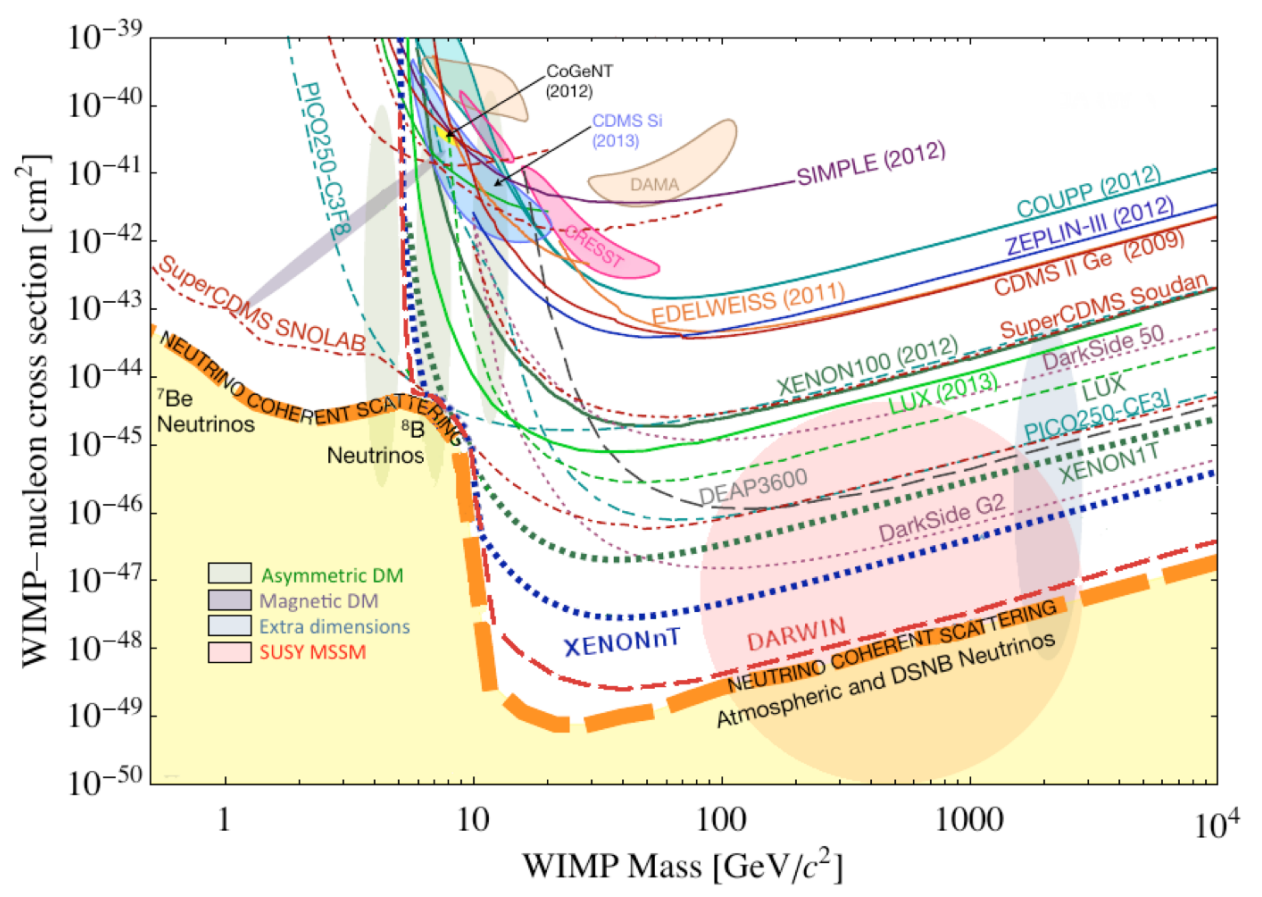

Figure 7: Experimentally accessible parameter space for WIMPs. The solid curves represent WIMPnucleon spin-independent cross section limits, the dot and dot-dashed curves show projected sensitivities, and hints for WIMP signals are indicated with shaded closed contours. An approximate band at the bottom represents the region where the sensitivity of direct dark matter experiments to WIMPs is limited by coherent scattering of ${ }^{8} \mathrm{~B}$ solar neutrinos, atmospheric neutrinos, and diffuse supernova neutrinos with nuclei. Figure adapted from [23].

The XENON collaboration successfully operates liquid xenon TPCs for direct WIMP searches, and has obtained the world's best limits on spin-independent WIMP nucleon cross sections with the XENON10 [5, 10] and XENON100 [6, 7] detectors. The current stage of the project, XENON100, is still being operated at LNGS. A data acquisition period with a total lifetime of $\sim 150$ days was recently completed, extending the detector operation to the fifth year and increasing the sensitivity of the search for an annual modulation. The analysis of these data is in an advanced stage and unblinding is expected within a couple of months. A study of the XENON100 response to single electrons has been published recently [24], improving our understanding of the dual-phase xenon-based TPC, and enabling searches for low energy events (i.e. WIMP-electron interactions for WIMP masses below $1 \mathrm{GeV} / \mathrm{c}^{2}$ ) based on S2 signal alone. Another paper submitted recently presents the first results on searches for axions and axion-like-particles with the XENON100 experiment [25]. 
The next phase in the XENON dark matter search program is the XENON1T experiment. The detector contains $3 \mathrm{t}$ of LXe surrounded by a $9.6 \mathrm{~m}$ diameter water Cerenkov shield, which has already been assembled in Hall B of LNGS. The inner vessel will house the liquid xenon, the TPC and two arrays of photomultiplier tubes (PMTs). The TPC will be made of interlocking PTFE panels, and the drift field homogeneity will be achieved with equidistant OFHC field shaping rings. The photosensors will be arranged in two arrays, containing 127 PMTs above the target in the gas phase, and 121 PMTs at the bottom of the sensitive liquid xenon volume. An upgrade of the TPC (XENONnT detector) is foreseen, which will allow for rather easy scaling of the target mass to $\sim 7 \mathrm{t}$ while keeping the same subsystems in place, including the water shield, cooling and support structure, xenon storage and purification, distillation column, DAQ and cabling, and the outer cryostat.

The R\&D work and design of the ultimate DARWIN experiment have started, with the goal to build a multi-purpose detector capable of constraining the mass and cross section of dark matter particles (in case of detection), of measuring the solar pp-neutrinos in real-time and of searching for the neutrino less double beta decay of ${ }^{136} \mathrm{Xe}$. A detailed Monte Carlo study of the sensitivity of a $21 \mathrm{t}$ xenon-based detector within the DARWIN project to different physics channels was recently published in Ref. [22].

\section{References}

[1] A. Kish, PhD thesis, University of Zurich, Switzerland (2011), http://opac.nebis.ch/ediss/20121322.pdf.

[2] R.E. Shanu, Nucl. Instr. Meth. 14, 297 (1961).

[3] M. Suzuki, Port. Phys. 12, 9 (1981).

[4] R.A. Muller et al., Phys. Rev. Lett. 27, 532 (1971).

[5] J. Angle et al. (XENON10 collaboration), Phys. Rev. Lett. 100, 021303 (2008).

[6] E. Aprile et al. (XENON collaboration), Phys. Rev. Lett. 107, 131302 (2011).

[7] E. Aprile et al. (XENON collaboration), Phys. Rev. Lett. 109, 181301 (2012).

[8] E. Aprile et al. (XENON collaboration), Astropart. Phys. 35, 573 (2012).

[9] E. Aprile et al. (XENON10 collaboration), Astropart. Phys. 34, 679-698 (2011).

[10] J. Angle et al. (XENON10 collaboration), Phys. Rev. Lett. 101, 091301 (2008).

[11] E. Aprile et al. (XENON collaboration), Astropart. Phys. 35, 34-49 (2011).

[12] M. Yamashita et al., NIM A 535, 692 (2004).

[13] E. Aprile et al., IEEE Trans. Nucl. Sci. 51, 1986 (2004).

[14] D.S. Akerib et al. (LUX collaboration), Phys. Rev. Lett. 112, 091303 (2014).

[15] C. Strege et al., JCAP 04, 013 (2013).

[16] O. Buchmueller et al., Eur. Phys. J. C 72, 2243 (2012).

[17] A. Fowlie et al., Phys. Rev. D 86, 075010 (2012).

[18] E. Aprile et al. (XENON collaboration) arXiv:1406.2374 (2014). 
[19] L. Baudis et al., JINST 8, P04026 (2013).

[20] T. Haruyama et al., Adv. Cryog. Eng. 710, 1459 (2004).

[21] L. Baudis (DARWIN consortium), J. Phys. Conf. Ser. 375, 012028 (2012).

[22] L. Baudis et al., JCAP 01, 044 (2014).

[23] P. Cushman et al., arXiv:1310.8327 (2014).

[24] E. Aprile et al. (XENON collaboration), J. Phys. G: Nucl. Part. Phys. 41, 035201 (2014).

[25] E. Aprile et al. (XENON collaboration), arXiv:1404.1455 (2014). 